\title{
Editorial: Academic Advising and Tutoring for Student Success in Higher Education: International Approaches
}

\author{
Emily A. McIntosh ${ }^{1 *}$, Liz Thomas ${ }^{2}$, Wendy G. Troxel ${ }^{3}$, Oscar van den Wijngaard ${ }^{4}$ and \\ David Grey ${ }^{5}$ \\ ${ }^{1}$ Middlesex University, London, United Kingdom, ${ }^{2}$ Edge Hill University, Ormskirk, United Kingdom, ${ }^{3}$ Kansas State University, \\ Manhattan, KS, United States, ${ }^{4}$ Maastricht University, Maastricht, Netherlands, ${ }^{5}$ UK Advising and Tutoring, York, \\ United Kingdom
}

Keywords: academic advising, personal tutoring in higher education, student success, student transition, student support

Editorial on the Research Topic

Academic Advising and Tutoring for Student Success in Higher Education: International Approaches

Research in advising and tutoring in Higher Education (HE) is mostly focused on the US context and, although this literature is widely available, it lacks a systematic, logical path to discovery and access (Troxel et al., 2021). This research topic is aligned with a content analysis of global advising literature, led by Dr. Wendy Troxel at NACADA, which will help to address this issue and identify new avenues of enquiry, focusing in particular on strengthening the evidence-base for advising practice. In an international context, we believe advising and tutoring to be of great importance and, in that regard, global research will help to build a credible evidence base for our practice and to acknowledge the centrality of high quality advising and tutoring to teaching, learning and student success. The promotion of personalized, even individualized, learning is now at the heart of most $\mathrm{HE}$ institutional missions, and many universities are currently reviewing their strategic and operational tutoring infrastructure (Lochtie et al., 2018; Thomas, 2012, Thomas, 2017). Indeed, agendas align worldwide to both expand and diversify HE and there is a global focus on understanding the factors that drive student persistence, student outcomes and employability, as well as the context of the role within HE. Personal tutoring has been proven to play a pivotal role creating a bridge between individual students and large and potentially anonymous institutions (Thomas and Hixenbaugh, 2006; Thomas, 2017). In the wake of the Covid19 pandemic, these personal connections need to be emphasized and it is vital that we continue to address known disparities in HE, improve access and participation and redress the marginalization of certain student groups and identities within institutions and disciplines. We believe that effectively tackling these issues will require strategic, connected, purposeful and effective ways of working across recognized institutional and international boundaries.

This significant shift in the national and international learning, teaching and student experience cannot be addressed without due regard to the strategic placement of advising and tutoring within universities, in a bid to continuously improve HE learning cultures. Moreover, approaches will increasingly have to understand the challenges and opportunities afforded to us by online instruction and the realization of the blended model of learning and teaching. Recently, Effective Personal Tutoring in Higher Education was published (Lochtie et al., 2018), promoting a more evidence-based approach to advising and tutoring practice and situating it within the wider, international literature 
on student retention and success. Additionally, two academic journals have long focused on scholarship related to academic advising (the NACADA Journal and The Mentor), though until fairly recently the audience has been primary focused within the United States of America (USA).

The range of venues for publishing advising scholarship is increasing. Nevertheless, there are still many gaps in our knowledge and many fruitful avenues for future research in this area, all of which can help to acknowledge and articulate the ongoing importance of advising and tutoring to fostering independence of thought and enthusiasm for lifelong learning. It is our intention, in coordinating this collection of articles, to bring together global scholarship on advising and connecting its varied themes. We can learn much by embracing international approaches to research and best practice in advising, and we must also do more to understand the impact of COVID19 on educational experiences and outcomes. An ongoing content analysis project undertaken by the NACADA Center for Research at Kansas State University outlines the themes and trends in advising and tutoring research and identifies emerging issues and avenues for enquiry in this important area. Most notably, the number of articles related to the use of technology in advising and tutoring was rising before COVID19 forced widespread use of synchronous and asynchronous online education.

The aim of this collection of articles is to further stimulate discussion in this field, whilst considering some of the most pressing gaps in the current literature, promoting further international research in this area and connecting several disparate HE policymaking agendas. The collection highlights the impact of high-quality advising and tutoring practices and is intent on advancing evidence that advising and tutoring are fundamental to helping universities achieve their strategic ambitions for student success. This final collection features 14 articles, edited by 5 guest associate editors and 44 manuscript authors. The articles span United States, United Kingdom, UAE, Australian and European advising and tutoring contexts. Many of the articles reflect the successful partnerships established by a number of organisations invested in the development of international approaches to advising and tutoring. This includes NACADA: The Global Community for Academic Advising (NACADA), UK Advising and Tutoring (UKAT), LVSA, the Dutch national association of academic advisors and the John $\mathrm{N}$ Gardner Institute for Excellence in Undergraduate Education.

This collection addresses several broad themes within the area of advising and tutoring. The collection brings to life all the ways in which advising and tutoring impacts student success more broadly, aligning approaches with student voice and partnership and co-design principles. Dominant themes within the collection reflect recent research and practice and include: 1) the consideration of skills and competencies of advisors, professional standards, and the design and development of digital training resources, 2) the impact of advising on student engagement, student voice, partnership and student transition, 3) the application of various tools and techniques in advising and tutoring practice, 4) the impact of advising and tutoring on attainment, mental health and student wellbeing, and 5) the use of technology, learning analytics and online spaces in advising and tutoring. All of these themes address how advising can help tackle known disparities in HE, improve access and participation and redress the marginalization of certain student groups. In that regard, this collection of articles will doubtless help inform future research enquiry focused on the impact of advising and tutoring on specific groups of vulnerable students in HE.

Within the theme of skills, competencies and professional standards, McGill et al. (Kansas State University, United States, The American University of Sharjah UAE and the University of Birmingham, United Kingdom) discuss the skills and competencies for effective academic advising and personal tutoring. Their article reflects approaches across three international advising and tutoring contexts and examines evidence of professional values, professional skills, professional behaviors, and training and continuing professional education and development. This article aligns with Walker's (Manchester Metropolitan University, United Kingdom) research article which considers the development and implementation of the UKAT Professional Framework for Advising and Tutoring. Continuing the theme of development for advisors, Woods' article (University of Warwick, United Kingdom) extends the evidence for professional skills, competencies, and standards by considering the development and design of an interactive digital training resource for personal tutors. This paper outlines the context of the training's development, and the pedagogic approaches, methods and principles that informed the learning design. Millard (Abertay University, United Kingdom) and Janjua's (Birmingham City University, United Kingdom) Policy and Practice Review considers the role of other stakeholders in advising, examining the role of Student Success Advisors (SSAs) as a targeted and specific aspect of support and advising for students that focuses upon student transition and the first-year experience (Millard and Janjua).

Within the theme of the impact of advising on student engagement, student voice, partnership and student transition, Yale (Edge Hill University, United Kingdom) considers the application of the psychological contract in advising. This research article examines the usefulness of psychological contract theory to explore the student-personal tutor relationship from the student perspective. The findings reveal the vital role the personal tutor has in the making, shaping, and negotiating of the student's psychological contract, which goes beyond the bounds of that specific relationship to the contract students have with the institution. Similarly, Raby's (University of Lincoln, United Kingdom) report considers the student voice in personal tutoring, examining students' own perspectives of the advising and tutoring process, intended to inform enhancements to the process. Goldspink and Engward's (Anglia Ruskin University, United Kingdom) article continues the analysis of staff student partnership by revisiting the tutorial as academic care. They present a phenomenological study of distance learning students, providing transferable insights about the immediate and lasting impact of the tutorial relationship. Continuing with this theme of student voice and partnership in advising, Mann's 
(University of Melbourne, Australia) article focusses on the cocreation and co-design of advising services, and examines how these systems can be built to address the central challenge of supporting learning outcomes, improving the student experience, and enhancing the acquisition of employability skills. Finally, Partington's (Kingston University, United Kingdom) conceptual analysis furthers our understanding of the student-advisor relationship and considers the development of personal tutoring as a key aspect of learner-centric pedagogy, in response to the changing profile of $\mathrm{HE}$ students, especially in terms of social and cultural capital.

Within the broad themes of the application of various tools and techniques in advising and tutoring practice and the emerging field of learner analytics, Lowes' (Plymouth University, United Kingdom) article examines the use of the Johari Window and the application of learning analytics. The article complements Yale's use of the psychological contract in advising, and argues that learning analytics systems have the potential to facilitate communication and sharing of information, and thus enhance the quality of communication between personal tutors and their tutees to improve student engagement and support the tutee. Learning analytics are also a strong feature of Ahern's (University College London, United Kingdom) article, which investigates the alignment of learning analytics and student wellbeing in the United Kingdom, providing data for advisors to identify changes in students' behaviour. Similarly, Holland et al. (Sheffield Hallam University, United Kingdom) study explores the extent of the relationship between advising and attainment

\section{REFERENCES}

Bhabba, H. (1994). The location of Culture. London, United Kingdom: Routledge. Gutierrez, K. (2008). Developing a sociocultural literacy in the third space. Read. Res. Q. 43, 148-164. doi:10.1598/rrq.43.2.3

Lochtie, D., McIntosh, E., Stork, A., and Walker, B. (2018). Effective personal tutoring in higher education. Cheshire, United Kingdom: Critical Publishing.

Thomas, L. (2012). What works? Student retention and success programme report. York, United Kingdom: Higher Education Academy.

Thomas, L. (2017). What works 2, student retention and success programme final report. York, United Kingdom: Higher Education Academy.

Thomas, L., and Hixenburgh, P. (2006). Personal tutoring in higher education. Stoke-on-Trent, United Kingdom: Trentham Books. and offers an institutional case study focused on the topic. Finally, McIntosh et al. (Middlesex University, London, United Kingdom, The Ohio State University, United States and UK Advising and Tutoring) Perspectives piece brings the collection together by considering learning analytics from a flipped advising standpoint, examining the concept of the "third space" (Bhabba, 1994; Gutierrez 2008) and extending the concept of advisor-student partnership. The "Third Space" is not just a physical space, rather a term used to define spaces where hybrid identifications are possible and where cultural transformations can happen.

Whilst this special collection of articles presents a variety of contemporary research agendas and perspectives in the field of advising and tutoring, we will continue this work into the future building on the knowledge and investigating further avenues of scholarly enquiry. Indeed, a collaborative approach to the identification and development of critical questions, support for research, dissemination of, and access to, this emerging body of scholarship is necessary. Discussions are underway to create an international repository of scholarship related to academic advising and personal tutoring. This database will further encourage scholars, practitioners, and decision-makers to situate this complex field within an established, growing body of literature.

\section{AUTHOR CONTRIBUTIONS}

Editorial coordinated by several guest editors of a research topic.

Troxel, W. G., Rubin, L., Grey, D., and McIntosh, E. A. (2021). Content analysis of 15 years of advising-related research in higher education, Research Report, NACADA Center for Research, Kansas State University, forthcoming.

Conflict of Interest: The authors declare that the research was conducted in the absence of any commercial or financial relationships that could be construed as a potential conflict of interest.

Copyright (c) 2021 McIntosh, Thomas, Troxel, van den Wijngaard and Grey. This is an open-access article distributed under the terms of the Creative Commons Attribution License (CC BY). The use, distribution or reproduction in other forums is permitted, provided the original author(s) and the copyright owner(s) are credited and that the original publication in this journal is cited, in accordance with accepted academic practice. No use, distribution or reproduction is permitted which does not comply with these terms. 